\title{
Desenvolvimento de Bebês Nascidos Pré-Termo e Indicadores Emocionais Maternos
}

\author{
Development of Preterm Infants and Mothers' Emotional Indicators
}

\author{
Daniele Abruzzi de Fraga, Maria Beatriz Martins Linhares*, \\ Ana Emília Vita Carvalho \& Francisco Eulógio Martinez \\ Universidade de São Paulo, Ribeirão Preto, Brasil
}

\begin{abstract}
Resumo
O objetivo do presente estudo longitudinal foi comparar o desenvolvimento de crianças nascidas pré-termo, em dois grupos diferenciados quanto à presença $(\mathrm{G} 1)$ ou ausência $(\mathrm{G} 2)$ de sintomas de ansiedade e depressão materna no período neonatal do bebê. A amostra foi composta por 32 crianças nascidas pré-termo, atendidas em seguimento psicológico desde a internação na UTI Neonatal até o final do primeiro ano de vida, sendo 11 do G1 e 21 do G2. Foram aplicados o Teste de Denver-II e a Escala do Desenvolvimento do Comportamento da Criança com as crianças, e o Inventário de Ansiedade Traço-Estado e o Inventário de Depressão Beck com as mães. As crianças de ambos os grupos apresentaram indicadores de desenvolvimento no primeiro ano de vida classificados como Normal, independentemente da presença de ansiedade e depressão materna.

Palavras-chave: Pré-termo; ansiedade materna; depressão materna.
\end{abstract}

\begin{abstract}
The aim of this longitudinal study was to compare the developmental outcomes of preterm infants allocated into two different groups taking into account the presence (G1) or lack (G2) of mothers' anxiety and depression symptoms during the neonatal period of the babies. The sample was based on 32 preterm infants who had a psychological follow up from the NICU internship period the end of first year of life. Group 1 included 11 infants and mothers with clinical anxiety and depression symptoms and Group 2 included 21 infants and mothers without clinical symptoms (control group). The infants were assessed by the Denver-II Test and Infant Scale of Development and Behavior and the mothers by the Inventory State- Trait Anxiety and Beck Depression Inventory. The children of both groups showed indicators of development on the first year of life classified as normal, regardless of the anxiety and depression symptoms of their mothers.

Keywords: Preterm; maternal anxiety; maternal depression.
\end{abstract}

A prematuridade e o baixo peso ao nascimento caracterizam-se como fatores de risco biológico para o desenvolvimento típico infantil, aumentando a probabilidade para problemas em diversas áreas e fases evolutivas (Chaudhari, Kulkarni, Pajnigar, Pandit \& Descmukh, 1991; Linhares, 2004). Outros fatores, tais como maior incidência de complicações neonatais e patologias, do tipo doença pulmonar crônica, displasia broncopulmonar, retinopatia, entercolite necrosante, apnéia e paralisia cerebral, podem associar-se à prematuridade (Cunha, Mezzacappa Filho \& Ribeiro, 2003; Hack \& Fanaroff, 2000).

Além dos riscos para a criança, o nascimento pré-termo de muito baixo peso ( $<1.500$ gramas) tem sido apontado como estressor ao equilíbrio emocional materno. Estudos mostram que, após o nascimento do bebê e durante sua

\footnotetext{
Endereço para correspondência: Universidade de São Paulo, Faculdade de Medicina de Ribeirão Preto, Laboratório de Pesquisa em Prevenção de Problemas de Desenvolvimento e Comportamento da Criança, Av. Tenente Catão Roxo, 2650, salas 52/53, Campus Universitário Monte Alegre, Ribeirão Preto, SP, 14048-900. Tel.: (16)3602 4610; Fax: (16) 36024504 E-mail:linhares@fmrp.usp.br; danieleabruzzi@ig.com.br
}

internação na Unidade de Tratamento Intensivo Neonatal (UTIN), as mães de bebês nascidos pré-termo apresentam alta incidência de sintomas de depressão (Poehlmann \& Fiese, 2001; Davis, Edwards, Mohay \& Wollin, 2003) e ansiedade elevada (Feldman, Weller, Leckman, Kuint \& Eidelman, 1999; Padovani, 2005).

Estes sintomas emocionais maternos têm sido apontados, por sua vez, como fatores de risco para o desenvolvimento da criança. A literatura indica associação entre sintomas de depressão nas mães e maior dificuldade na formação de apego seguro pela criança (Poehlmann \& Fiese, 2001), assim como entre estes sintomas e baixa responsividade das mães, com uma interação mãe-bebê prejudicada (Wilfong, Saylor \& Elksnin, 1991). A ansiedade materna também tem sido associada a comprometimento na formação de apego seguro pela criança (Feldman et al., 1999). Além disso, foi observado por Wijnroks (1999) que as mães com alto nível de ansiedade recordada do período neonatal eram mais ativas na interação com seus bebês prematuros, porém, mais intrusivas e menos responsivas com sensibilidade, aos seis meses, em situação lúdica semi-estruturada, quando comparadas 
a mães com pouca ansiedade. A atividade materna compreendia falar, brincar ou estimular a criança, ao passo que a intrusividade referia-se à super-estimulação, interferência, diretividade com comportamentos inadequados e sem sensibilidade, levando a criança a desviar a atenção e se afastar da atividade na interação com a mãe. A sensibilidade correspondia ao envolvimento da mãe, sem ser intrusiva e sem interferir com as atividades da criança.

Levando-se em conta de um lado, o risco da prematuridade para o equilíbrio emocional materno e, por outro, o risco da ansiedade e depressão das mães para o desenvolvimento de suas crianças, tem sido recomendada a implementação de programas de informação e suporte psicológico aos pais (Bracht, Ardal, Bot \& Cheng, 1998; Carvalho, 2005; Linhares et al., 2000; Wilfong et al., 1991), assim como avaliação do desenvolvimento em esquema longitudinal das crianças nascidas pré-termo, para verificação de riscos e adequada estimulação (Liaw \& Brooks-Gunn, 1993; Linhares et al., 2000; Pedromônico, 2001; Linhares, Carvalho, Machado \& Martinez, 2003; Nobre, 2003).

Alguns estudos mostram os efeitos positivos das intervenções de suporte e informações aos pais. Estas se associam com menor nível de estresse parental, redução nos escores de ansiedade do tipo estado e depressão materna (Preyde \& Ardal, 2003), presença de menos sentimentos negativos dos pais em relação à situação do nascimento prematuro, maior interação destes com seus bebês na UTIN (Bracht et al., 1998), redução no estresse materno e maior crença das mães nas suas habilidades em exercer a maternagem. Além disso, verifica-se associação com melhores indicadores de desenvolvimento mental das crianças aos três e seis meses de idade cronológica corrigida (Melnyk et al., 2001). O acompanhamento longitudinal do desenvolvimento das crianças também mostrou relação com melhores indicadores do desenvolvimento, na medida em que pode detectar precocemente problemas e oferecer orientações promotoras do desenvolvimento (Liaw \& Brooks-Gunn, 1993; Linhares et al., 2003).

Neste sentido, o presente estudo ${ }^{1}$ teve por objetivo comparar indicadores de desenvolvimento, no primeiro ano de vida, avaliados em grupos de bebês pré-termo, diferenciados quanto à presença de indicadores emocionais clínicos maternos.

\section{Método}

\section{Participantes}

A amostra foi composta por 32 bebês nascidos pré-termo com muito baixo peso $(<1500 g)$, no ano de 2001, no Setor de Neonatologia do Hospital das Clínicas da Facul-

${ }^{1}$ Parte da Monografia de Bacharelado em Psicologia (Faculdade de Filosofia, Ciências e Letras de Ribeirão Preto da Universidade de São Paulo - USP) da primeira autora sob orientação da segunda. Projeto aprovado pelo Comitê de Ética em pesquisa do Hospital de Clínicas da Faculdade de Medicina de Ribeirão Preto - USP, bolsista de Iniciação Científica da Fundação de Apoio à Pesquisa do Estado de São Paulo. dade de Medicina de Ribeirão Preto-Universidade de São Paulo (HCFMRP), que foram internados na Unidade de Tratamento Intensivo Neonatal (UTIN) após o nascimento, e suas respectivas mães. Esta foi extraída de um estudo mais amplo de Carvalho (2005) e Padovani (2005). Dos participantes em potencial para integrar o estudo $(n=71)$, foram excluídos 28 devido aos seguintes motivos: (a) os bebês cujas mães eram analfabetas $(n=4)$; (b) os bebês cujas mães eram portadoras do vírus HIV $(n=1)$ ou apresentavam antecedentes psiquiátricos $(n=6)$, considerando que estes fatores influenciam diretamente o quadro emocional materno e a interação mãe-bebê, podendo interferir nos resultados do estudo; (c) bebês que foram a óbito (17). Dos 43 restantes, 11 mães não aceitaram participar do estudo.

A amostra de conveniência de 32 bebês e suas mães foi distribuída em dois grupos de acordo com indicadores emocionais clínicos maternos. O primeiro grupo (G1) foi formado por 11 bebês e suas mães, sendo que estas apresentaram indicadores de ansiedade, depressão e/ou disforia por ocasião do período neonatal do bebê. O segundo grupo (G2), por sua vez, foi formado por 21 bebês e suas mães, sem indicadores emocionais clínicos (grupo de comparação).

\section{Instrumentos}

Para a realização deste estudo, foram utilizados os seguintes instrumentos: (a) IDATE - Inventário de Ansiedade Traço-Estado (Biaggio \& Natalício, 1979), para avaliação da ansiedade; (b) BDI - Inventário de Depressão Beck (Cunha, 2001), para avaliação de depressão e disforia; (c) SCID/Não Paciente - Entrevista Clínica Estruturada para o DSM III-R (Spitzer, Williams, Gibbon \& First, 1989), para detecção de antecedentes psiquiátricos; (d) EDCC - Escala do Desenvolvimento do Comportamento da Criança: O primeiro Ano de Vida (Pinto, Vilanova \& Vieira, 1997), que avalia o desenvolvimento da criança combinando três eixos relacionados: anatômico corporal (axial ou apendicular), estimulação (espontâneo ou estimulado) e função (não comunicativo ou comunicativo); (e) Teste de Triagem de Desenvolvimento de Denver II (Frankenburg et al., 1990/1999), que avalia o risco no desenvolvimento da criança em quatro grandes áreas: Pessoal-Social, Linguagem, Motor Fino-Adaptativo e Motor Grosseiro; (f) Ficha para Coleta de Informações dos Prontuários Médicos do HCFMRP dos bebês; (g) Ficha de Características Sócio-Demográficas.

\section{Procedimento}

Após a assinatura do Termo de Consentimento Livre e Esclarecido pelas mães, foi aplicado o SCID/Não Paciente a fim de identificar aquelas que apresentavam antecedentes psiquiátricos; estas foram excluídas do estudo. Posteriormente, foi iniciada a coleta de dados com o preenchimento de uma Ficha de Características Sócio-Demográficas dos participantes e a aplicação dos inventários de avaliação psicológica de ansiedade (IDATE) e depressão e disforia (BDI) nas mães. Na avaliação da ansiedade estado, pedia- 
se para a mãe focalizar a situação atual de internação do bebê. Para neutralizar a interferência da aplicação de um teste sobre o outro (efeito "carry-over"), foi realizado um balanceamento, alterando-se a ordem de aplicação de cada instrumento.

Com base nos resultados obtidos, a amostra foi distribuída nos dois grupos. Identificou-se a presença de indicadores clínicos de ansiedade materna com base no percentil igual ou acima de 75 no IDATE e de depressão e disforia, utilizando-se pontuação acima de 20 e de 15 no BDI, respectivamente, como sugerido para pacientes não diagnosticados (Gorenstein \& Andrade, 2000).

A avaliação psicológica das mães, com os mesmos instrumentos, foi realizada em outros dois momentos, a saber: após a alta hospitalar do bebê (junto ao primeiro retorno médico, realizado no primeiro mês após a alta), e no final do primeiro ano de vida da criança. Na avaliação da ansiedade estado, pedia-se para a mãe focalizar a situação atual, respectivamente da alta hospitalar e do primeiro ano de vida da criança.

Procedeu-se à consulta aos prontuários médicos, de enfermagem e do Serviço de Psicologia Pediátrica do HCFMRP, para obtenção de informações sobre sua saúde e desenvolvimento ao longo do primeiro ano de vida. Neste serviço, os bebês são acompanhados longitudinalmente com avaliações do desenvolvimento até a fase escolar e orientações às mães. Nas avaliações do primeiro ano de vida aplicam-se rotineiramente os testes Denver II e EDCC. Foram objeto de análise deste estudo as aplicações do Denver-II realizadas aos 5-7 e 10-11 meses e da EDCC aos 5-7 e 9-11 meses de idade cronológica corrigida (ICC) da criança. Deve-se salientar que, necessariamente, cada participante deveria ter no seu prontuário a avaliação do Setor de Psicologia, por meio do Denver II e/ou da EDCC. O desempenho no Denver II recebeu a atribuição 1 para risco e o para normal. O desempenho na EDCC recebeu a seguinte atribuição em escala ordinal: $1=$ atraso, $2=$ risco, $3=$ regular, $4=$ bom e $5=$ excelente.

A análise documental dos prontuários médicos foi realizada a fim de identificar a lista de problemas de saúde, a evolução clínica na UTIN e o índice de risco clínico neonatal, medido por meio do Índice de Risco Clínico para Bebês (Cockburn et al., 1993 - CRIB). Este instrumento analisa a presença de fatores de risco e estresse neonatal considerando-se os seguintes indicadores na história de saúde do bebê: peso ao nascimento, idade gestacional, fração mínima e/ou máxima do oxigênio inspirado, acidose sangüínea e malformações congênitas, avaliados nas primeiras 12 horas de vida do neonato.

Os dados do estudo foram quantificados em termos de freqüência, proporção ou incidência, dependendo de sua natureza. O tratamento estatístico incluiu a análise de comparação entre grupos (G1 X G2), por meio do Teste de Mann-Whitney ou o Teste do Qui-quadrado, dependendo da natureza da variável (contínua ou categórica) e a análise de comparação intra-grupo, por meio do Teste de Wilcoxon, considerando-se os indicadores emocionais maternos nos três diferentes momentos comparados dois a dois (internação do bebê $\mathrm{X}$ após a alta hospitalar; internação do bebê X final do primeiro ano; após a alta hospitalar X final do primeiro ano). A análise de dados foi processada pelo Statistical Package for Social Sciences (SPSS versão 12.0).

\section{Resultados}

\section{Características das Crianças}

Quanto ao sexo das crianças, $46 \%$ do G1 e $71 \%$ do G2 eram do sexo feminino, não havendo diferença significativa entre os dois grupos $(p=0,25)$. A mediana do peso ao nascimento das crianças de ambos os grupos foi de aproximadamente 1080 gramas e a idade gestacional por volta de 30 semanas. As medidas do índice de risco neonatal Apgar do quinto minuto e CRIB - indicaram que, em ambos os grupos, as crianças tinham uma condição de risco neonatal leve e bom prognóstico de evolução clínica. O tempo de internação das crianças teve grande amplitude de variação, aproximadamente de cinco a 70 dias na UTIN, com 30 a 135 dias de permanência no hospital. Os grupos foram semelhantes quanto às características das crianças e seu estado de saúde no período neonatal, não havendo diferença significativa entre os mesmos (Tabela 1).

Tabela 1

Características das Crianças do G1 e G2

\begin{tabular}{lccccc}
\hline & \multicolumn{2}{c}{ Mediana } & \multicolumn{2}{c}{ Valor mínimo-Valor máximo } & $\mathrm{z}$ \\
\cline { 2 - 6 } Características das crianças & $\begin{array}{c}\mathrm{G} 1 \\
(n=11)\end{array}$ & $\begin{array}{c}\mathrm{G} 2 \\
(n=21)\end{array}$ & $\begin{array}{l}\mathrm{G} 1 \\
(n=11)\end{array}$ & $\begin{array}{l}\text { G2 } \\
(n=21)\end{array}$ \\
\hline Peso ao nascimento (gramas) & 1080 & 1075 & $710-1410$ & $470-1425$ & $-0,18$ \\
Idade gestacional (semanas) & 30 & 31 & $28-34$ & $23-34$ & $-0,36$ \\
Índice de risco clínico neonatal & 2 & 2 & $0-9$ & $0-9$ & $-0,37$ \\
Apgar 5 min & 9 & 9 & $6-10$ & $5-10$ & $-0,20$ \\
Tempo total de internação (dias) & 69 & 59 & $34-146$ & $27-129$ & $-0,37$ \\
Tempo de internação na UTIN (dias) & 34 & 13 & $5-65$ & $6-92$ & $-0,45$ \\
\hline
\end{tabular}




\section{Indicadores do Ambiente Familiar}

Não houve diferença significativa entre os dois grupos nas variáveis referentes às características do ambiente familiar. A idade das mães na gravidez revelou predominância de mães jovens, com mediana de 25 anos e idade mínima de 13, em ambos os grupos. Em relação à escolaridade, tanto as mães (proporção: $\mathrm{G} 1=0,64 ; \mathrm{G} 2=0,62$ ) quanto os pais (proporção: $\mathrm{G}_{1}=0,73 ; \mathrm{G} 2=0,72$ ) tinham predominantemente nível de instrução do ensino fundamental ( $1^{\mathrm{a}}$ a $8^{\mathrm{a}}$ série) em ambos os grupos. No que diz respeito à ocupação, a maioria das mães não exercia atividades remuneradas fora de casa (proporção: $\mathrm{G} 1=0,64$; $\mathrm{G}_{2}=0,62$ ). Os pais, por sua vez, encontravam-se formalmente empregados em sua maioria (proporção: $G 1=0,82$; G2=0,86), em ambos os grupos. Quanto à qualificação profissional, em ambos os grupos, a maioria das atividades remuneradas exercidas pelos pais (proporção: $\left.\mathrm{G}_{1}=0,91 ; \mathrm{G}_{2}=0,76\right)$ e mães (proporção: $\mathrm{G}_{1}=0,75$;
G2=0,76) eram não qualificadas ou de qualificação inferior, segundo classificação de Soares e Fernandes (1989). As casas das famílias de ambos os grupos tinham em média cinco cômodos, sendo o número de moradores em média de quatro pessoas no G1 e cinco no G2.

\section{Indicadores Emocionais Maternos}

Houve diferença significativa entre os grupos quanto aos escores de ansiedade e depressão materna por ocasião do período neonatal, em que os bebês encontravam-se internados na UTIN; o G1 apresentou escores mais elevados de indicadores emocionais do que o G2. Observouse que, após a alta hospitalar, permaneceu a diferença entre os grupos no mesmo sentido dos dados encontrados no período neonatal. Porém, aos 12 meses de ICC das crianças, não houve diferença significativa entre os grupos quanto aos escores emocionais de ansiedade e depressão das mães (Tabela 2).

Tabela 2

Indicadores de Ansiedade e Depressão Materna nos Três Momentos Avaliados G1 e G2

\begin{tabular}{|c|c|c|c|c|c|}
\hline \multirow[b]{2}{*}{ Indicadores emocionais maternos } & \multicolumn{2}{|c|}{ Mediana } & \multicolumn{2}{|c|}{ Valor mínimo-Valor máximo } & \multirow[t]{2}{*}{$\mathrm{z}$} \\
\hline & $\mathrm{G} 1(n=11)$ & $\mathrm{G} 2(n=21)$ & $\mathrm{G} 1(n=11)$ & $\mathrm{G} 2(n=21)$ & \\
\hline & \multicolumn{4}{|c|}{ Durante a internação do bebê na UTIN } & \\
\hline Ansiedade estado & 87 & 33 & $32-99$ & $\mathrm{O}-73$ & $-4,11^{* * *}$ \\
\hline Ansiedade traço & 55 & 27 & $19-96$ & $0-48$ & $-3,15^{* * *}$ \\
\hline \multirow[t]{2}{*}{ Depressão } & 17 & 5 & $4-38$ & $0-12$ & $-3,64^{*} *$ * \\
\hline & \multicolumn{4}{|c|}{ Após a alta hospitalar do bebê } & \\
\hline Ansiedade estado & 64 & 3 & $\mathrm{O}-99$ & $0-73$ & $-3,21$ ** \\
\hline Ansiedade traço & 39 & 11 & $5-98$ & $0-55$ & $-3,01$ ** \\
\hline \multirow[t]{2}{*}{ Depressão } & 13 & 3 & $0-24$ & $0-14$ & $-2,47$ 米米 \\
\hline & \multicolumn{4}{|c|}{ No primeiro ano de vida da criança } & \\
\hline Ansiedade estado & 35 & 2 & $\mathrm{O}-8 \mathrm{O}$ & $0-54$ & $-1,63$ \\
\hline Ansiedade traço & 35 & 30 & $0-87$ & $0-81$ & $-0,52$ \\
\hline Depressão & 8 & 7 & $0-22$ & $0-21$ & $\mathrm{O}$ \\
\hline
\end{tabular}

Nota. ${ }^{*} \mathrm{pd} \leq 0,05 ;{ }^{*} * \mathrm{pd} \leq 0,01$.

Quanto aos escores de ansiedade estado, do tipo situacional, estes diminuíram significativamente em ambos os grupos do momento de internação do bebê para o momento após a alta hospitalar ( $\mathrm{z}=-2,8 \mathrm{O}, p=0, \mathrm{O} 1 \mathrm{em} \mathrm{G1}$; $\mathrm{z}=-2,18, p=0,03$ em G2). Os valores encontrados neste último momento não diferiram significativamente dos encontrados por ocasião do primeiro ano de vida da criança em ambos os grupos. Quanto aos escores de ansiedade traço, referente a características mais estáveis da personalidade do indivíduo, estes diminuíram significativamente nas mães do G1 do período de internação do bebê em comparação com o final do primeiro ano de vida $(z=-2,03$; $p=0,04)$. No G2, por sua vez, não houve diferença significativa entre os escores de ansiedade traço nas mães entre os momentos avaliados.
Quanto aos escores de depressão, houve redução significativa apenas no G1, do período de internação para os momentos após a alta hospitalar do bebê $(z=-2,81 ; p=0,01)$ e no final do primeiro ano de vida $(\mathrm{z}=-2,37 ; p=0,02)$, respectivamente.

\section{Indicadores de Saúde no Período Neonatal}

No que diz respeito à evolução clínica das crianças, não houve diferença significativa entre os grupos quanto à incidência das doenças mais freqüentes na amostra total, cuja incidência foi maior do que 0,20. Os três problemas de saúde mais freqüentes foram: desconforto respiratório (incidência: $\mathrm{G}_{1}=0,82 ; \mathrm{G} 2=0,67$ ), apnéia (incidência: $\mathrm{G} 1=0,64 ; \mathrm{G} 2=0,48)$ e retinopatia da prematuridade (incidência: $\left.\mathrm{G}_{1}=0,45 ; \mathrm{G}_{2}=0,33\right)$. 


\section{Indicadores de Desenvolvimento - Denver-II}

Foram avaliadas pelo Denver-II 10 crianças do G1 e 16 crianças do G2 aos 5-7 meses de ICC e oito crianças do G1 e 13 crianças do G2 aos 10-11 meses de ICC. Os indicadores de risco para problemas do desenvolvimento, medidos por meio do Denver-II, revelaram que menos da metade das crianças de ambos os grupos avaliados apresentaram risco no desenvolvimento aos 5-7 meses de ICC (proporção: G1=0,10; G2=0,25) e 10-11 meses de ICC (proporção: $\mathrm{G} 1=0,25 ; \mathrm{G} 2=0,46)$. Não houve diferença significativa entre os grupos quanto aos indicadores avaliados pelo Denver-II nas duas faixas etárias.

\section{Indicadores do Desenvolvimento - EDCC}

Foram avaliadas pela EDCC nove crianças do G1 e 14 crianças do G2 aos 5-7 meses de ICC e seis crianças do G1 e cinco crianças do G2 aos 9-11 meses de ICC. Aos 57 meses de ICC, mais da metade das crianças de ambos os grupos tiveram seu desenvolvimento classificado entre bom e excelente em todas as áreas do comportamento medidas pelo instrumento. Nesta idade, os indicadores de risco ou atraso apresentaram-se em torno de $20 \%$, variando de zero a $28 \%$ (Tabelas 3 e 4 ).

Tabela 3

Indicadores do Desenvolvimento Não Comunicativo Medidos pela EDCC aos 5-7 e 9-11 Meses de ICC

\begin{tabular}{|c|c|c|c|c|}
\hline \multirow[b]{2}{*}{ Áreas da EDCC } & \multicolumn{2}{|c|}{$\begin{array}{l}\text { Proporção } \\
\text { (5-7 meses) }\end{array}$} & \multicolumn{2}{|c|}{$\begin{array}{l}\text { Proporção } \\
\text { (9-11 meses) }\end{array}$} \\
\hline & G1 $(n=9)$ & $\mathrm{G}_{2}(\mathrm{n}=14)$ & G1 $(n=6)$ & $\mathrm{G}_{2}(\mathrm{n}=5)$ \\
\hline \multicolumn{5}{|c|}{ Axial espontâneo não comunicativo } \\
\hline Atraso & 0,11 & 0,21 & $\mathrm{O}$ & 0,20 \\
\hline Risco & $\mathrm{O}$ & 0,07 & $\mathrm{O}$ & $\mathrm{O}, 2 \mathrm{O}$ \\
\hline Regular & 0,11 & 0,07 & 0,17 & 0,20 \\
\hline Bom & 0,78 & 0,58 & 0,66 & 0,20 \\
\hline Excelente & $\mathrm{O}$ & 0,07 & 0,17 & $\mathrm{O}$ \\
\hline Dado prejudicado & $\mathrm{O}$ & $\mathrm{O}$ & $\mathrm{O}$ & 0,20 \\
\hline \multicolumn{5}{|c|}{ Axial estimulado não comunicativo } \\
\hline Atraso & $\mathrm{O}$ & 0,15 & - & - \\
\hline Risco & 0,11 & $\mathrm{O}$ & - & - \\
\hline Regular & 0,11 & 0,31 & - & - \\
\hline Bom & 0,45 & 0,08 & - & - \\
\hline Excelente & 0,33 & 0,46 & - & - \\
\hline \multicolumn{5}{|c|}{ Apendicular espontâneo não comunicativo } \\
\hline Risco & $\mathrm{O}$ & 0,21 & $\mathrm{O}$ & $\mathrm{O}$ \\
\hline Regular & 0,22 & $\mathrm{O}$ & $\mathrm{O}$ & $\mathrm{O}$ \\
\hline Bom & 0,67 & 0,79 & 0,50 & 0,80 \\
\hline Excelente & $\mathrm{O}$ & $\mathrm{O}$ & 0,50 & 0,20 \\
\hline Dado prejudicado & 0,11 & $\mathrm{O}$ & $\mathrm{O}$ & $\mathrm{O}$ \\
\hline \multicolumn{5}{|c|}{ Apendicular estimulado não comunicativo } \\
\hline Atraso & $\mathrm{O}$ & 0,07 & $\mathrm{O}$ & $\mathrm{O}$ \\
\hline Risco & 0,22 & 0,14 & $\mathrm{O}$ & $\mathrm{O}$ \\
\hline Regular & $\mathrm{O}$ & 0,07 & $\mathrm{O}$ & $\mathrm{O}$ \\
\hline Bom & 0,56 & 0,58 & 0,83 & $1, \mathrm{OO}$ \\
\hline Excelente & 0,11 & 0,14 & 0,17 & $\mathrm{O}$ \\
\hline Dado prejudicado & 0,11 & $\mathrm{O}$ & $\mathrm{O}$ & $\mathrm{O}$ \\
\hline
\end{tabular}

Nota. As atividades relacionadas ao campo Axial estimulado não comunicativo não se aplicam para a idade de 9-11 meses. 
Tabela 4

Indicadores do Desenvolvimento Comunicativo Medidos pela EDCC aos 5-7 e 9-11 Meses de ICC

\begin{tabular}{|c|c|c|c|c|}
\hline \multirow[b]{2}{*}{ Áreas da EDCC } & \multicolumn{2}{|c|}{$\begin{array}{c}\text { Proporção } \\
\text { (5-7 meses) }\end{array}$} & \multicolumn{2}{|c|}{$\begin{array}{c}\text { Proporção } \\
\text { (9-11 meses) }\end{array}$} \\
\hline & G1 $(n=9)$ & G2 $(n=14)$ & $\mathrm{G} 1(\mathrm{n}=6)$ & $\mathrm{G}_{2}(\mathrm{n}=5)$ \\
\hline & \multicolumn{4}{|c|}{ Axial espontâneo comunicativo } \\
\hline Atraso & $\mathrm{O}$ & $\mathrm{O}, \mathrm{O} 7$ & 0,33 & 0,20 \\
\hline Regular & $\mathrm{O}$ & $\mathrm{O}$ & 0,17 & $\mathrm{O}$ \\
\hline Bom & $1, \mathrm{OO}$ & 0,79 & 0,50 & 0,80 \\
\hline \multirow[t]{2}{*}{ Excelente } & $\mathrm{O}$ & 0,14 & $\mathrm{O}$ & $\mathrm{O}$ \\
\hline & \multicolumn{4}{|c|}{ Axial estimulado comunicativo } \\
\hline Bom & 0,78 & 0,67 & 0,67 & 0,40 \\
\hline \multirow[t]{2}{*}{ Excelente } & 0,22 & 0,33 & 0,33 & 0,60 \\
\hline & \multicolumn{4}{|c|}{ Apendicular espontâneo comunicativo } \\
\hline Atraso & $\mathrm{O}$ & $\mathrm{O}$ & - & - \\
\hline Risco & 0,11 & 0,08 & - & - \\
\hline Bom & 0,67 & 0,75 & - & - \\
\hline Excelente & 0,11 & 0,17 & - & - \\
\hline \multirow[t]{2}{*}{ Dado prejudicado } & 0,11 & $\mathrm{O}$ & - & - \\
\hline & \multicolumn{4}{|c|}{ Apendicular estimulado comunicativo } \\
\hline Atraso & $\mathrm{O}$ & $\mathrm{O}$ & 0,33 & $\mathrm{O}$ \\
\hline Regular & $\mathrm{O}$ & $\mathrm{O}$ & 0,17 & $\mathrm{O}$ \\
\hline Bom & 0,89 & 0,91 & 0,33 & $1, \mathrm{OO}$ \\
\hline Excelente & $\mathrm{O}$ & 0,09 & 0,17 & $\mathrm{O}$ \\
\hline Dado prejudicado & 0,11 & $\mathrm{O}$ & $\mathrm{O}$ & $\mathrm{O}$ \\
\hline
\end{tabular}

Nota. As atividades relacionadas ao campo Apendicular espontâneo comunicativo não se aplicam para a idade de 9-1 1 meses.

Aos 9-11 meses, por sua vez, pelo menos metade das crianças de ambos os grupos obtiveram classificação entre bom e excelente nas áreas avaliadas. Fez exceção o campo Axial espontâneo não comunicativo, que inclui itens como "mantém em pé com o mínimo de apoio", "caminha com auxílio", "dá alguns passos sem apoio" e "caminha independentemente”. Neste, no G2, 60\% das crianças apresentaram resultados entre as classificações de atraso, risco ou regular.

Embora a maior parte dos resultados mostre classificações na EDCC adequadas, deve-se comentar que os riscos ou atrasos observados em torno de 30\% incidiram nas seguintes áreas: no G1, nos campos Axial espontâneo comunicativo e Apendicular estimulado comunicativo, aos 9-11 meses de ICC e, no G2, no campo Axial espontâneo não comunicativo aos 5-7 e 9-11 meses de ICC. Não houve diferença significativa entre os dois grupos (G1 X G2) nas duas faixas etárias estudadas em relação aos indicadores de desenvolvimento medidos pela EDCC, nas seguintes áreas: Axial espontâneo não comunicativo (5-7 meses: $\mathrm{z}=-0,45 ; p=0,73$ e 9-11 meses: $\mathrm{z}=-1,94 ; p=0,07)$, Axial estimulado não comunicativo ( $5-7$ meses: $\mathrm{z}=-0,03 ; p=0,84)$, Apendicular espontâneo não comunicativo (5-7 meses: $\mathrm{z}=-0,09 ; p=0,97$ e 9-11 meses: $\mathrm{z}=-0,98 ; \mathrm{p}=0,43)$, Apendicular estimulado não comunicativo (5-7 meses: $\mathrm{z}=-$ $0,07 ; p=0,97$ e 9-11 meses: $\mathrm{z}=-0,91 ; p=0,76)$, Axial espon- tâneo comunicativo (5-7 meses: $\mathrm{z}=-0,48 ; p=0,78$ e $9-11$ meses: $\mathrm{z}=-1,06 ; p=0,43)$, Axial estimulado comunicativo (5-7 meses: $\mathrm{z}=-0,43$; $p=0,54$ e 9-1 1 meses: $\mathrm{z}=-0,84 ; p=0,54)$, Apendicular espontâneo comunicativo (5-7 meses: $z=-0,64$; $p=0,79)$ e Apendicular estimulado comunicativo (5-7 meses: $z=-0,76 ; p=0,79$ e $9-11$ meses: $z=-1,06 ; p=0,43)$.

\section{Discussão}

Os achados do presente estudo mostraram que os dois grupos de crianças foram semelhantes quanto às características neonatais, patologias freqüentes na evolução clínica e indicadores de seu ambiente familiar. Em ambos os grupos, as crianças tinham o peso ao nascimento com mediana por volta de 1080 gramas, portanto um peso extremamente baixo, e idade gestacional com mediana por volta de 30 semanas. Essas características indicam uma condição de risco biológico para o desenvolvimento típico infantil (Chaudhari et al., 1991; Linhares, 2004). Apesar da ocorrência das condições de prematuridade e muito baixo peso ao nascimento das crianças, os indicadores de gravidade neonatal, medidos pelos índices do CRIB e do Apgar do $5^{\circ}$ minuto, revelaram que estas apresentavam risco leve, com classificação de bom prognóstico para sua evolução clínica. 
Em relação à evolução propriamente dita, as crianças de ambos os grupos apresentaram alta freqüência de patologias, principalmente respiratórias. Dentre as doenças com maior incidência (acima de 40\%), destacaram-se o desconfor to respiratório, apnéia e retinopatia. Esses achados confirmam a literatura que aponta a prematuridade e o muito baixo peso ao nascimento como fatores que aumentam a probabilidade de ocorrência de patologias ao longo do desenvolvimento (Cunha et al., 2003; Hack \& Fanaroff, 2000), com alta incidência de patologias relacionadas ao sistema respiratório (Cunha et al., 2003) e retinopatia da prematuridade (Hack \& Fanaroff, 2000). Deve-se observar que a associação entre prematuridade e incidência de patologias na história de vida da criança aumenta o risco de prejuízo para o seu desenvolvimento (Bordin, 2005; Chaudhari et al., 1991; Ohlweiler, Silva, Barros, Riesgo \& Rotta, 2003; Stoelhorst et al., 2003).

Quanto aos indicadores emocionais maternos, detectouse diferença significativa entre os grupos nos momentos de internação do bebê na UTIN e após a alta hospitalar. Os indicadores emocionais de ansiedade dos tipos traço e estado e depressão foram mais elevados no G1 do que no G2, nos dois momentos referidos. De acordo com a literatura, as emoções maternas de ansiedade (Feldman et al., 1999; Padovani, Linhares, Carvalho, Duarte \& Martinez, 2004) e depressão (Davis et al., 2003; Poehlmann \& Fiese, 2001) constituem-se como fatores de risco para o desenvolvimento da criança. Assim, no G1, além do risco biológico devido à condição de prematuridade, muito baixo peso e incidência de patologias, verificou-se um fator de risco adicional referente ao estado emocional materno. Como salientado por Linhares, Bordin e Carvalho (2004), diversos fatores de risco presentes na vida de um mesmo indivíduo tendem a se multiplicar, antes que apenas somar-se, de tal forma que um agrava o outro, aumentando a ameaça ao desenvolvimento sadio e adaptativo.

Quanto à comparação entre os indicadores emocionais maternos nos três momentos avaliados, verificou-se que, tanto no G1 quanto no G2, houve redução significativa nos escores de ansiedade do tipo estado, ou seja, situacional, do período de internação do bebê ao momento da al ta hospitalar e ao final do primeiro ano de vida, respectivamente. Essa redução pode estar relacionada ao suporte psicológico e de informações que é oferecido às mães no Programa de Seguimento Longitudinal (PSL) de prematuros do HCFMRP. A existência de programas de suporte e de informação aos pais está associada com menor nível de estresse parental, redução nos escores de ansiedade estado e depressão (Preyde \& Ardal, 2003), menos sentimentos negativos dos pais em relação ao nascimento prematuro, maior interação destes com seus bebês na UTIN (Bracht et al., 1998), redução no estresse materno e maior crença das mães nas suas habilidades para exercer a maternagem (Melnyk et al., 2001).

Além da redução do nível de ansiedade estado, ligada ao período do nascimento prematuro e internação do bebê, no G1 houve redução significativa nos escores de depressão e ansiedade traço do momento de internação ao final do primeiro ano de vida. Pode-se, assim, supor que, neste período, as mães conseguiram se reorganizar do ponto de vista emocional.

Com relação ao desenvolvimento das crianças, verificouse que estas apresentaram bons indicadores no primeiro ano de vida, medidos pelo Denver II e EDCC, ao contrário do que poderia ser esperado pela sua condição de vulnerabilidade. Essa condição adequada de desenvolvimento no primeiro ano de vida de grande parte da amostra estudada pode estar relacionada ao acompanhamento que as crianças e as mães recebem no PSL do HCFMRP. Este programa é rotina para os bebês egressos da UTIN e conta com o apoio e promoção do desenvolvimento psicológico das crianças, tendo como objetivo minimizar os efeitos negativos da prematuridade no desenvolvimento. Como aponta a literatura, o acompanhamento longitudinal do desenvolvimento de crianças nascidas prematuras e com muito baixo peso está associado a melhores indicadores do seu desenvolvimento, na medida em que detecta precocemente problemas e oferece orientações promotoras do desenvolvimento (Liaw \& Brooks-Gunn, 1993; Linhares et al., 2003).

Aos 5-7 meses de ICC, a proporção de crianças, de ambos os grupos, com o desenvolvimento classificado em risco pelo Denver II e em risco ou atraso pela EDCC, não ultrapassou a porcentagem de $28 \%$. Embora a maior parte dos resultados mostrem classificações adequadas na EDCC aos 5-7 meses, os atrasos e riscos observados nesta idade incidiram principalmente no G2, no campo Axial espontâneo não comunicativo (30\%). Este resultado confirma os achados de Nobre (2005) e Linhares et al. (2003), que verificaram que, por volta dos seis meses, as crianças nascidas pré-termo e com muito baixo peso tinham maior prejuízo no desenvolvimento motor em comparação às outras áreas do seu desenvolvimento.

Quanto ao fato de o maior prejuízo no desenvolvimento motor amplo ter sido verificado nas crianças do G2, pode-se especular que a presença de sintomas emocionais clínicos maternos, predominantemente de ansiedade, funcionou como fator de proteção ao desenvolvimento motor amplo das crianças do G1. Neste caso, as mães mais ansiosas podem ter interagido com seus bebês de forma mais ativa, como foi observado por Wijnroks (1999) em situação lúdica semi-estruturada, podendo estimular suas crianças e proporcionar o desenvolvimento motor com menos prejuízos. Deve-se ressaltar, no entanto, a relevância de se considerar o nível de ansiedade ótimo para estimulação. Se, por um lado, a maior responsividade e diretividade materna no início do desenvolvimento de crianças vulneráveis, tendem a influenciar positivamente seu desenvolvimento (Landry, Smith, Swank \& MillerLoncar, 2000), por outro a intrusividade torna-se restritiva de oportunidades ao desenvolvimento da criança, limitando sua autonomia.

Houve uma elevação na proporção de crianças em risco, avaliado pelo Denver- II aos 10-11 meses de ICC, e em risco ou atraso, medidos pela EDCC aos 9-1 1 meses de ICC, mas esta não ultrapassou a metade das crianças, em am- 
bos os grupos. Nesta idade, os indicadores de risco e atraso concentraram-se nos campos Axial espontâneo comunicativo e Apendicular espontâneo comunicativo, no G1, e Axial espontâneo não comunicativo no G2. Esses achados estão de acordo com alguns estudos que também encontraram incidência de prejuízo no desenvolvimento motor (Chaudhari et al., 1991; Linhares et al., 2003) e da linguagem (Linhares et al., 2003; Nobre, 2005) no final do primeiro ano de vida.

Quanto à maior incidência, no $\mathrm{G} 1$, de atraso e risco nas áreas referentes à linguagem, podemos supor que os níveis mais elevados de ansiedade e depressão materna tenham tido um efeito negativo na interação dessas mães com seus bebês, influenciando seu desenvolvimento. As mães ansiosas podem ter interagido com seus filhos de forma mais intrusiva, como observado por Wijnroks (1999) em situação lúdica semi-estruturada, estimulando excessivamente, o que desvia a atenção da criança da atividade, dificultado o exercício e desenvolvimento da linguagem. As mães depressivas, por sua vez, tendem a interagir com seus bebês de forma menos responsiva (Wilfong et al., 1991), o que pode ter gerado um ambiente menos estimulador para as crianças do G1.

Os grupos foram mais semelhantes do que diferentes em seus indicadores de desenvolvimento no primeiro ano de vida. Este achado indica que o início do desenvolvimento das crianças vulneráveis estudadas parece não ter sido fortemente associado aos indicadores emocionais maternos avaliados (ansiedade, predominantemente). Cabe ressaltar que as mães das crianças da amostra também receberam na UTIN acompanhamento psicológico para ajudar no enfrentamento da situação de ansiedade e estresse devido à internação do bebê. Esse fato, como aponta a literatura, pode contribuir para criar um contexto de suporte à saúde mental materna e atenuar os possíveis efeitos adversos dos fatores emocionais de ansiedade das mães e, conseqüentemente, favorecer o desenvolvimento dos bebês (Bracht et al., 1998; Carvalho, 2005; Melnyk et al., 2001; Preyde \& Ardal, 2003).

Finalizando, cabe ressaltar algumas limitações do presente estudo. Trata-se de uma amostra pequena e do tipo de conveniência, que foi selecionada em contexto privilegiado de suporte interdisciplinar aos bebês prematuros e familiares de um hospital universitário de nível terciário, o qual é referência como centro de excelência no Sistema Único de Saúde. Essa característica específica pode ter um efeito indireto nos achados, na medida em que cria um contexto positivo de desenvolvimento para a criança, com apoio às mães no período de internação do bebê na UTIN e no seguimento longitudinal.

Destaca-se que, na amostra estudada, havia uma complexa condição de risco biológico e psicológico quando se analisam as condições do bebê e da saúde mental materna. Porém, os achados sobre o desenvolvimento do bebê foram otimistas em relação a níveis mais altos de comprometimento encontrados em grupos com o risco estabelecido da prematuridade. $\mathrm{O}$ apoio psicológico às mães $\mathrm{e}$ crianças pode ter atuado como mecanismo de proteção aos efeitos negativos do risco, o que pode justificar os resultados mais favoráveis.

Os achados do presente estudo reforçam a necessidade de haver na área da Saúde programas de avaliação do desenvolvimento das crianças nascidas prematuramente e do estado emocional materno, a fim de assegurar medidas de prevenção em grupos de crianças vulneráveis devido aos riscos neonatais, por meio de programas de followup interdisciplinar.

\section{Referências}

Biaggio, A. M. B., \& Natalício, L. (1979). Inventário de ansiedade traço-estado. Rio de Janeiro, RJ: CEPA.

Bordin, A. B. M. (2005) Desenvolvimento psicológico na fase escolar de crianças ex-prematuras, diferenciadas quanto ao risco clínico neonatal. Tese de Doutorado não-publicada, Universidade de São Paulo, Ribeirão Preto, SP.

Bracht, M., Ardal, F., Bot, A., \& Cheng, C. M. (1998). Initiation and maintenance of a Hospital-Based parent group for parents of premature infants: Key factors for success. Neonatal Network, 17(3), 291-311.

Carvalho, A. E. V. (2005). Indicadores emocionais maternos e intervenção psicológica durante a internação do bebê pré-termo em UTI Neonatal. Tese de Doutorado não-publicada, Universidade de São Paulo, Ribeirão Preto, SP.

Chaudhari, S., Kulkarni, S., Pajnigar, F., Pandit, A. N., \& Descmukh, S. A. (1991). Longitudinal follow-up of development of preterm infants. Journal of the Indian Academy of Pediatrics, 28(8), 873-880.

Cockburn, F., Cooke, R. W. I., Gamsu, H. R., Greenough, A., Hopkins, A., Mcintosh, N. et al. (1993). The CRIB (Clinical Risk Index for Babies) score: A tool for assessing initial neonatal risk and comparing performance of neonatal intensive care units. Lancet, 342, 193-198.

Cunha, J. A. (2001). Manual da versão em português das escalas Beck. São Paulo, SP: Casa do Psicólogo.

Cunha, G. S., Mezzacappa, F., Filho, \& Ribeiro, J. D. (2003). Fatores maternos e neonatais na incidência de displasia bronco pulmonar em recém-nascidos de muito baixo peso. Jornal de Pediatria, 79(6), 550-556.

Davis, L., Edwards, H., Mohay, H., \& Wollin, J. (2003). The impact of very premature birth on the psychological health of mothers. Early Human Development, 73, 61-70.

Feldman, R., Weller, A., Leckman J. F., Kuint, J., \& Eidelman, A. I. (1999). The nature of the mother's tie to her infant: Maternal bonding under conditions of proximity, separation, and potential loss. Journal Child Psychological Psychiatric, 40(6), 929939.

Frankenburg, W. K., Doods, J. B., Archer, P., Brernick, B., Mashka, P., Edelman, N. et al. (1999). Teste de triagem de desenvolvimento de Denver II. (M. R. Pedromônico, E. L. Bragatto \& R. Strobilius, Trads.). (Original publicado 1990)

Gorenstein, C., \& Andrade, L. (2000). Inventário de depressão de Beck: Propriedades psicométricas da versão em português. In C. Gorenstein, L. H. S. G. Andrade \& A. W. Zuardi (Eds.), Escalas de avaliação clínica em psiquiatria e psicofarmacologia (pp. 89-95). São Paulo, SP: Lemos.

Hack, M., \& Fanaroff, A. A. (2000). Outcomes of children of extremely low birthweight and gestational age in the 1990s. Seminars in Neonatology, 5(2), 89-106. 
Landry, S. H., Smith, K. E., Swank, P. R., \& Miller-Loncar, C. L. (2000). Early maternal and child influences on children's later independent cognitive and social functioning. Child Development, $71(2)$, 358-375.

Liaw, R. F., \& Brooks-Gunn, J. (1993). Patterns of low-birthweight children's cognitive development. Developmental Psychology, 29(6), 1024-1035.

Linhares, M. B. M. (2004). Estresse, resiliência e cuidados no desenvolvimento de neonatos de alto risco. In E. G. Mendes, M. A. Almeida \& L. C. A. Williams (Eds.), Temas em educação especial (pp. 315-324). São Carlos, SP: Editora da Universidade Federal de São Carlos.

Linhares, M. B. M., Bordin, A. B. M., \& Carvalho, A. E. V. (2004). Aspectos do desenvolvimento psicológico da criança ex-prematura na fase escolar. In E. M. Marturano, M. B. M. Linhares \& S. R. Loureiro (Eds.), Vulnerabilidade e proteção: Indicadores na trajetória de desenvolvimento do escolar (pp. 75-106). São Paulo, SP: Casa do Psicólogo.

Linhares, M. B. M., Carvalho, A. E. V., Bordin, M. B. M., Chimmelo, J., T., Martinez, F. E., \& Jorge, S. M. (2000). Prematuridade e muito baixo peso como fatores de risco ao desenvolvimento da criança. Cadernos de Psicologia e Educação - Paidéia, 10(12), 60-69

Linhares, M. B. M., Carvalho, A. E. V., Machado, C., \& Martinez, F. E. (2003). Desenvolvimento de bebês nascidos pré-termo no primeiro ano de vida. Cadernos de Psicologia e Educação Paidéia, 13(25), 57-72.

Melnyk, B. M., Alpert-Gills, L., Feinstein, N. F., Fairbanks, E., Schultz-Czarniak, J., Hust, D. et al. (2001). Improving cognitive development of low-birth-weight premature infants of COPE program: A pilot study of the benefit of early NICU intervention with mothers. Research in Nursing e Health, 24, 373-389.

Nobre, F. D. A. (2005). Indicadores do desenvolvimento de crianças nacidas pré-termo de muito baixo peso nos dois primeiros anos de vida. Dissertação de Mestrado não-publicada, Universidade de São Paulo, Ribeirão Preto, SP.

Ohlweiler, L., Silva, A. R, Barros, S. V., Riesgo, R., \& Rotta, N. T. (2003). Influência da hemorragia intra-craniana e crises neonatais sobre o desenvolvimento neurológico e psicomotor de recém-nascidos prematuros no Hospital das Clínicas de Por to Alegre, Brasil. Arquivos de Neuro-Psiquiatria, 61(4), 902905.

Padovani, F. H. P. (2005). Indicadores emocionais de ansiedade, disforia e depressão e verbalizações maternas acerca do bebê, da amamentação e da maternidade em mães de bebês nascidos pré-termo de muito baixo peso, durante a hospitalização do bebê e após a alta, comparadas a mães de bebês nascidos a termo. Tese de Doutorado não-publicada, Universidade de São Paulo, Ribeirão Preto, SP.

Padovani, F. H. P., Linhares, M. B. M., Carvalho, A. E. V., Duarte, G., \& Martinez, F. E. (2004). Avaliação de sintomas de ansiedade e depressão em mães de neonatos pré-termo durante e após a hospitalização em UTI-Neonatal. Revista Brasileira de Psiquiatria, 26(4), 251-254.

Pedromônico, M. (2001). Avaliando o desenvolvimento. Arquivos de Neuropsiquiatria, 59, 16-17.

Pinto, E. B., Vilanova, L. C. P., \& Vieira, R. M. (1997). O desenvolvimento do comportamento da criança no primeiro ano de vida: Padronização de uma escala para a avaliação e o acompanhamento. São Paulo, SP: Casa do Psicólogo.
Poehlmann, J., \& Fiese, B. H. (2001). The interaction of maternal and infant vulnerabilities and developing attachment relationships. Development and Psychopathology, 3, 1-11.

Preyde, M., \& Ardal, F. (2003). Effectiveness of a parent "buddy" program for mothers of very preterm infants in a neonatal intensive care unit. Canadian Medical Association Journal, 61, 101-106

Soares, N. E., \& Fernandes, L. M. (1989). A medida do nível sócio-econômico-cultural. Arquivos de Psicologia, 41(2), 35-43.

Spitzer, R. L., Williams, J. B.W., Gibbon, M., \& First, M. B. (1989). Instruction manual for the structured clinical interview for DSMIII-R (SCID). New York: State Pediatric Institute.

Stoelhorst, G. M. S. J., Rijken, M., Martens, S. E., Zwieten, P. H. T., Feenstra, J., Zwinderman, H. et al. (2003). Developmental outcome at 18 and 24 months of age in very preterm children: A cohort study from 1996 to 1997. Early Human Development, 72, 83-95.

Wijnroks, L. (1999). Maternal recollected anxiety and motherinfant interaction in preterm infants. Infant Mental Health Journal, 20(4), 393-409.

Wilfong, E. W., Saylor, C., \& Elksnin, N. (1991). Influences on responsiveness: Interactions between mothers and their premature infants. Infant Mental Health Journal, 12(1), 31-40.
Recebido: 30/03/2006 $1^{a}$ revisão: 14/09/2006 $2^{a}$ revisão: $07 / 02 / 2007$ $3^{a}$ revisão: 21/06/2007 Aceite final: 27/06/2007 